\title{
Design of a Hexagonal Printed Monopole Antenna with Circular Slot for UWB Breast Cancer Imaging Applications
}

\author{
Salamatu Abubakar Ibrahim ${ }^{1}$, Isah Musa Danjuma ${ }^{1}$, Mohamed Abdul-Al², and Raed \\ Abd Alhameed ${ }^{1,2}$ \\ \{s.a.ibrah2@bradford.ac.uk, I.M.Dasnjuma@bradford.ac.uk, M.Abdul- \\ Al@bradford.ac.uk, and R.A.A.Abd@bradford.ac.uk\} \\ ${ }^{1}$ School of Electrical Engineering and Computer Science, University of Bradford, Bradford \\ BD71DP, United Kingdom \\ ${ }^{2}$ Faculty of Engineering and Informatics, University of Bradford, Bradford, BD7 1DP, UK.
}

\begin{abstract}
A low profile hexagonal printed monopole antenna with circular slot is presented for ultra-wide band (UWB) breast cancer imaging application. The sensor was designed to operate in the UWB band frequency range of 3.1-10.6 $\mathrm{GHz}$. The antenna dimensions are $30 \times 30 \times 1.6 \mathrm{~mm}^{3}$. FR4 substrate is used between ground and radiating patch having a relative permittivity of 4.6, and loss tangent of 0.0025. The antenna is designed using Computer Simulation Technology Software (CST). The simulation results reveals that the antenna bandwidth can be increased using the slots configurations.
\end{abstract}

Keywords: Microwave imaging, UWB antenna, monopole antenna, Breast cancer detection.

\section{Introduction}

Antenna design with the use of ultra-wideband communications has garnered a lot of considerations by researchers all over the world. These recognitions are due to low cost, low power consumption, small size, wide impedance bandwidth, omnidirectional characteristics, easy fabrication, and high data transmission rate [1]. These inherent qualities have made monopole antenna a good candidate for this research. Additionally, it could also be used for many communication applications such as radar application, position and tracking systems, microwave imaging application particularly for breast cancer imaging application [2].

By making certain changes to the antenna construction, monopole antennas are now being used for ultra-wideband technology. The technique for expanding bandwidth of ultra-wide band antennas by using slot methods of various shapes and configurations in the patch of the antenna, such as rectangular, partially circular, triangular, and hexagonal, is extensively considered for investigating impedance matching [3]. The effects of the slots on impedance bandwidth evaluation, radiation pattern characteristics, gain performance, and radiation efficiency were 
discovered by simulation testing.

Non-ionizing and non-destructive testing are two advantages of using a microwave imaging sensor as a medical imaging tool for breast cancer screening. In comparison to $\mathrm{x}$-ray mammography, microwave imaging has advantages of cheap cost, low complexity, high data rate, and no breast compression [4]. The antenna is employed as a sensor in microwave imaging to transmit and receive microwave signals into the breast tissues.

Changes in electrical characteristics of various tissues, such as relative permittivity and conductivity, provides the basis for this theory. The antenna's dispersed signal is utilized to identify the difference in dielectric characteristics between normal and malignant tissue in a more efficient, effective, safe, and precise manner. The difference in water content between normal and malignant tissues is used to detect the tumor in this approach. Microwaves can distinguish between healthy and unhealthy tissue. The presence of strong water within the malignant tissues is used as a tumor diagnostic factor. As a result, imaging using microwave antennas is another option for early breast cancer diagnosis, potentially saving a lot of lives. For microwave imaging measurements, a variety of UWB antennas have been proposed, including the bow tie and Vivaldi antennas [5, 6].

In the near field of the breast phantom, the use of a monopole antenna for the microwaveimaging provides a very effective and efficient performance [7]. Over the UWB band, antenna basics such as return loss, VSWR, and radiating pattern are well defined.

In this paper, a novel, low profile, low cost, enhanced impedance bandwidth and highperformance monopole antenna for breast cancer microwave imaging is presented. The antenna is small and uniform in size suitable for the microwave imaging application. In this study, the antenna was analysed, and the simulation results indicate good signal transmission and reception. The remaining of the paper are as follows: Section II presents the antenna geometry and optimization design, while Section III analyses the antenna performance measurements and conclusion are drawn in Section IV.

\section{Antenna Geometry And Optimisation Design}

\subsection{Antenna Geometry}

The proposed antenna has a uniform geometry of $30 \times 30 \mathrm{~mm}^{2}$ on a FR-4 substrate with thickness of $1.6 \mathrm{~mm}$, permittivity of 4.6, and loss tangent of 0.0025 . The basic antenna structure consists of a hexagonal patch with a circular slot, a feed line and 2 uniform rectangular planes acting as a partial ground. The hexagonal patch parameters were defined as follows: Substrate length (SL), Substrate width (SW), Patch length (PL) Microstrip length (ML), Microstrip width (MW), short side (SS), Long side (LS), radius (R), and angle ( $\left.\mathrm{a}^{0}\right)$, as shown in Fig.1 and Fig. 2 respectively. The values of UWB antenna parameters are tabulated in Table1. These parameters were optimized to get the optimum result used for the microwave imaging sensor and the antenna was fed with a $50 \Omega$ coaxial transmission. 


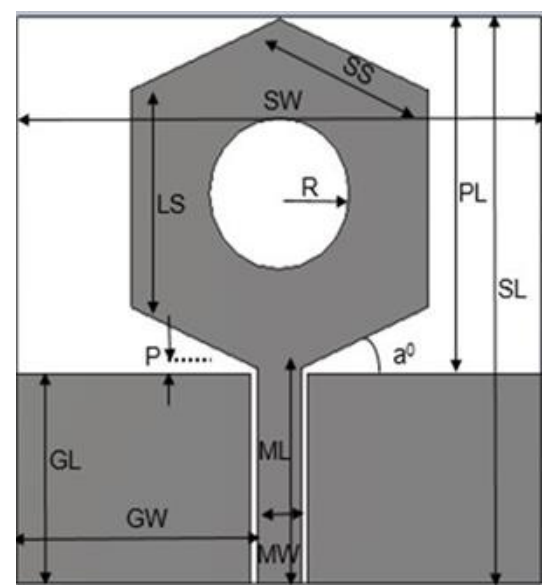

Fig.1. Geometry layout of the hexagonal monopole antenna.

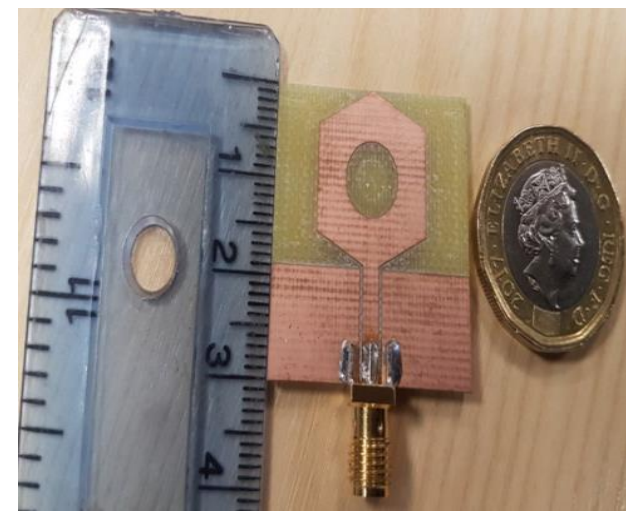

Fig.2. Physical implementation of the hexagonal monopole.



Fig.3. Far field Measurement of the proposed antenna in the anechoic chamber. 
Table 1: Dimensions specification of the proposed antenna

\begin{tabular}{|c|c|}
\hline Parameter & Dimensions in mm \\
\hline Substrate width (SW) & 30 \\
\hline Substrate length (SL) & 30 \\
\hline Substrate thickness (ST) & 1.6 \\
\hline Microstrip length (ML) & 11.40 \\
\hline Microstrip width (MW) & 2.50 \\
\hline Microstrip gap (MG) & 0.4 \\
\hline Long Side (LS) & 11.49 \\
\hline Short Side (SS) & 9.30 \\
\hline Radius (R) & 4 \\
\hline Angle (a $\left.{ }^{0}\right)$ & 25 \\
\hline Partial ground length (GL) & 11.10 \\
\hline Partial ground width (GW) & 13.35 \\
\hline
\end{tabular}

\subsection{Optimization Design}

All engineering designs require optimization to enable engineers obtain the greatest outcomes from the parameters of their designs. Small changes in a system's size can have a multiplier effect on the system overall efficiency. Interactions can be time-consuming in a system with numerous variables, making it impracticable to determine their optimal value. Computer simulation technology (CST) was utilized for this design, and the optimization was done with the particle swam optimisation method.

The algorithm takes the advantage of S-parameter sensitivity information used to reduce the number of simulations needed and speed up the optimization process [8]. It is the most robust of the optimization algorithms. The parameters that affect the performance of the proposed monopole UWB antenna are the gap between the 2 uniforms rectangular antenna planes acting as the partial ground, the partial ground length (GL and the angle at the bend of the corners of the ground plane $\left(\mathrm{a}^{0}\right)$. These parameters affect the proposed antenna radiation pattern, return loss and the impedance matching and hence, optimized to achieve better antenna performance as indicated in the return loss of Fig. 4 and 5 respectively.

\section{Antenna Performance Measurements}

The simulation results of the hexagonal monopole antenna have been analysed and optimized using CST microwave studio. Mat lab software was used to plot the simulated result, and Agilent technologies N5242 was used to acquire the measured results with the use of a vector network analyzer that covers the $10 \mathrm{MHz}$ to $26 \mathrm{GHz}$ frequency range. The simulated measurement is shown in Fig.4, while the simulated and measured return loss results are indicated in Fig.5.

The proposed antenna's $-10 \mathrm{~dB}$ operational bandwidth spans the UWB spectrum from 3.1 to 10.6 GHz. The first resonance occurs at $3.99 \mathrm{GHz}$, the second at $6.5 \mathrm{GHz}$, and the maximum resonance occurs at $10.6 \mathrm{GHz}$. The lower frequency bandwidth is significantly influenced by 
the slot in the monopole antenna's patch.

The current flow will follow the curvature of the slot if the slot is etched on the radiating patch. As a result, the slot shape has an impact on the distribution of surface current as indicated in Fig. 6. The course of the surface current is controlling the antenna's radiation [9]. Although, the proposed antenna design is simple and compact in size, it achieves the overall impedance bandwidth of the UWB spectrum, and the simulated and measured results are in good agreements.

The breast phantom electrical properties were taken from [4] and the modelling of skin and fat phantom were taken from CST's material library. The skin and fat have the following dimensions: skin with radius $82 \mathrm{~mm}$, thickness of $2 \mathrm{~mm}$ and depth of $32 \mathrm{~mm}$, fat with radius 80 $\mathrm{mm}$ and depth of $32 \mathrm{~mm}$. The plastic layer was modelled around the breast phantom as it was used in [5] to support the antenna. The plastic layer has a dimension including relative permittivity of $\varepsilon_{\mathrm{r}}=3$, conductivity of $\sigma=0.0015 \mathrm{~s} / \mathrm{m}$, radius $=92 \mathrm{~mm}$, thickness $=8 \mathrm{~mm}$, and depth of $32 \mathrm{~mm}$.

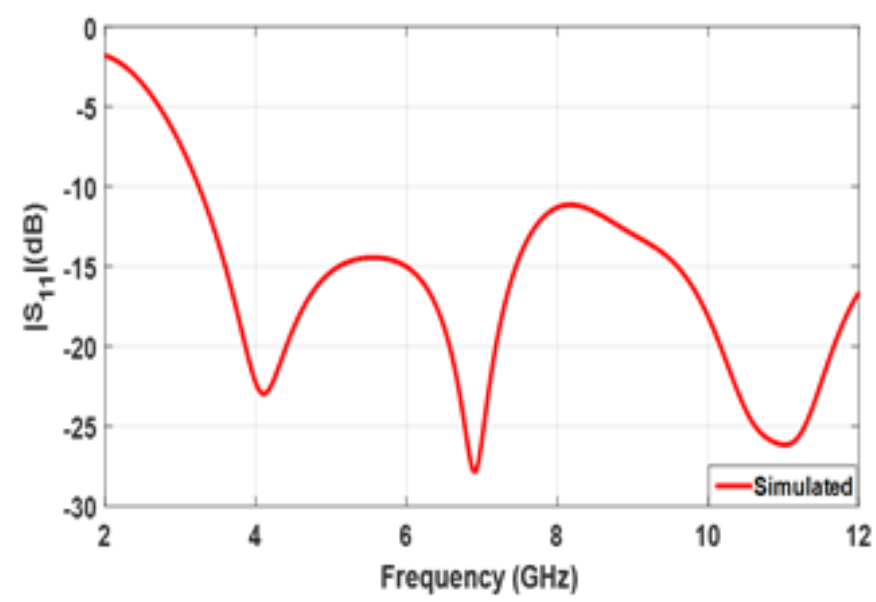

Fig.4. Simulated results with optimized parameters of gap $4 \mathrm{~mm}$ and partial ground length GL $=11.10$ $\mathrm{mm}$ and angle of $25^{\circ}$. 




Fig. 5. Simulated and measured results of the proposed antenna.
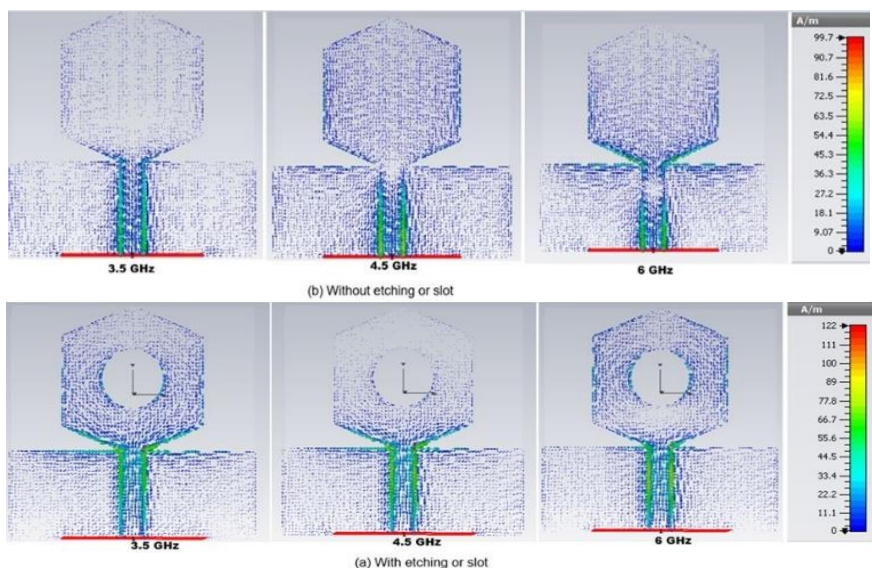

Fig.6. Surface current densities of the Proposed antenna.

\section{Conclusion}

An experimental investigation of a small UWB antenna was carried out for breast cancer microwave imaging. The antenna's low profile and simple construction make it an excellent option for microwave imaging applications. The excellent result between the simulated and measured results of the antenna is used to assess antenna performance. The antenna's uniform size of $30 \times 30 \mathrm{~mm} 2$ is suitable for use in a wide range of microwave imaging applications. 


\section{References}

[1] Haraz, O. and A.-R. Sebak, UWB antennas for wireless applications. Advancement in microstrip antennas with recent applications, 2013: p. 125-152.

[2] Adnan, S., et al., Simulation and experimental measurements for near field imaging. Progress In Electromagnetics Research, 2012.p. 433.438.

[3] Mohamed S. Soliman., et al., The Effect of the Ground Slots up on the Bandwidth Performance for UWB Antenna, in $18^{\text {th }}$ Mediterranean Microwave Symposium,2018: p.227-230.

[4] Mirza, A.F., et al., An active microwave sensor for near field imaging. IEEE Sensors Journal, 2017. 17(9): p. 2749-2757.

[5] I.M Danjuma, et al., Design and optimization of a bowtie dipole adjacent to dielectric material for through the wall imaging using hybrid electromagnetic computational analysis techniques. in 2017 Internet Technologies and Applications (ITA). 2017. IEEE.

[6] Danjuma, I., et al., Design of a taper slot low profile Vivaldi antenna for ultra-wideband microwave breast imaging applications. Journal of Multidisciplinary Engineering Science and Technology (JMEST).

[7] Danjuma, I.M., et al., AT slot monopole antenna for UWB microwave imaging applications. 2018.

[8] Liu, B., et al., Efficient global optimisation of microwave antennas based on a parallel surrogate model-assisted evolutionary algorithm. IET Microwaves, Antennas \& Propagation, 2018.

[9] Yaghjian, A. et al., Equivalence of surface current and aperture field integrations for reflector antennas. IEEE Transactions on antennas and propagation, 1984. 32(12): p. 1355-1358. 